\title{
EDUCAÇÃO INTERCULTURAL. TEORIAS, POLÍTICAS E PRÁTICAS DE PLURALISMO CULTURAL NO SISTEMA DE ENSINO ITALIANO
}

\author{
Marco Catarci ${ }^{1}$
}

\begin{abstract}
No contexto italiano, a pesquisa no âmbito da educação e a prática intercultural estão estruturadas, essencialmente, ao longo de dois eixos principais: a) em primeiro lugar, uma abordagem de educação intercultural visa identificar, planejar e experimentar as estratégias mais adequadas para promover uma inserção efetiva dos alunos estrangeiros na escola e na sociedade. Isso também exige a consecução das condições para assegurar que todos os indivíduos (autóctones e imigrantes) alcancem os mesmos níveis de sucesso escolar; b) em segundo lugar, uma vez que a educação intercultural se estende a todos e, especialmente, aos autóctones, assumir essa perspectiva significa atuar na tarefa fundamental de incentivar hábitos de acolhida entre os italianos. Isso pode e deve ter consequências na revisão e na reformulação do sistema educacional, que não deve estar voltado somente para a formação do cidadão italiano, mas, acima de tudo, do cidadão do mundo, que vive e trabalha em um contexto global interdependente.
\end{abstract}

Palavras-chave: educação intercultural, Itália, estudantes migrantes, interculturalidade, inclusão.

\section{Introdução. Educação e pluralismo cultural ${ }^{2}$}

A abordagem intercultural no contexto educacional define um projeto intencional de promoção do diálogo e do intercâmbio cultural direcionado a todos, autóctones e estrangeiros. Desta forma, as diversidades (cultural, de gênero, de classe social, biográficas, etc.) tornam-se um ponto de vista privilegiado dos processos educacionais, proporcionando oportunidades para que cada um se desenvolva a partir do que é.

\footnotetext{
1 Departamento de Ciências da Formação da Università degli Studi Roma Tre. Roma, Itália.

2 Artigo traduzido do italiano pela Equipe do CSEM.
} 
Uma perspectiva "multiculturalista" se desenvolveu na década de sessenta e setenta do século XX no Canadá e na Austrália e, em seguida, nos Estados Unidos para abordar a questão do gerenciamento das diferenças culturais no interior do Estado3: de forma específica, em 1971, o Canadá foi o primeiro país a adotar o multiculturalismo como uma política oficial, afirmando o valor e a dignidade dos cidadãos de qualquer pertença cultural, religiosa ou linguística.

Na Europa, uma perspectiva intercultural se desenvolveu em decorrência do aumento dos fluxos migratórios em países como França, Alemanha, Reino Unido, Bélgica e Países Baixos. As primeiras estratégias interculturais tiveram como foco medidas destinadas a filhos de trabalhadores migrantes para a aprendizagem da língua dos países de acolhida e para a manutenção daquela de origem, a fim de permitir seu regresso à pátria, tomando a forma de uma "pedagogia para estrangeiros" (ausländerpädagogik), na Alemanha, ou de uma "pedagogia de acolhida" (pédagogie d'accueil) na França ${ }^{4}$.

Na Itália, a educação intercultural também surgiu em decorrência do desenvolvimento do fenômeno imigratório, que ocorreu a partir dos meados dos anos setenta do século passado, porém com um atraso em relação aos países europeus de antiga imigração (França, Alemanha, Reino Unido). Em particular, o ano de 1973 é para a Itália o ano da "virada", onde se registra pela primeira vez um ligeiro "saldo migratório" positivo, referente à diferença entre o número de pessoas que entraram e que saíram do país.

No entanto, deve-se notar que a Itália não é hoje um país multicultural somente em decorrência do fenômeno migratório, mas também como resultado de outros processos históricos, que deram início a significativas relações de confronto, intercâmbio e conflito entre diferentes grupos culturais: a histórica presença das minorias linguísticas e culturais no país (com doze grupos linguísticos minoritários reconhecidos, que incluem aproximadamente dois milhões e meio de pessoas); a experiência do colonialismo (1882-1960), especificamente, na Líbia, Somália, Etiópia e Eritreia; o desenvolvimento do racismo desde a promulgação do Manifesto da raça (1938) e das Leis raciais (1938-39)5; e a longa história da emigração italiana (1876-1988), que levou para o exterior, nesse período, 27 milhões de compatriotas.

3 BARN, Ravinder. Interculturalism in Europe: Fact, fad or fiction - the deconstruction of a theoretical idea, p. 103.

4 PORTERA, Agostino. Pedagogia interculturale in Italia e in Europa, p. 482.

5 Sobre estas questões, cf. AA.VV. Manifesto della Razza; DEL BOCA, Angelo. Italiani in Africa Orientale. Vol.1-3; BEVILACQUA, Piero, DE CLEMENTI, Andreina, FRANZINA, Emilio (a cura di). Storia dell'emigrazione italiana. Vol. I - Partenze. Vol. II - Arrivi; DEL BOCA, Angelo. Italiani, brava gente? Un mito duro a morire; LABANCA, Nicola. Oltremare. Storia dell'espansione coloniale italiana; CAMPANI, Giovanna. Dalle minoranze agli immigrati. La questione del pluralismo culturale e religioso in Italia; COLUCCI, Michele. Lavoro in movimento. L'emigrazione italiana in Europa, 1945-57; LORÉ, Michele. Antisemitismo e razzismo ne La difesa della razza, 1938-1943. 
O fenômeno da imigração, ao contrário, apareceu na Itália desde os meados dos anos setenta do século passado, embora tenha alcançado em um intervalo de tempo relativamente curto dimensões quantitativas comparáveis aos países europeus de mais antiga tradição migratória.

Conforme o Istat, os imigrantes presentes atualmente na Itália são cinco milhões, ou seja, 8,3\% do total da população ${ }^{6}$. A rápida mudança demográfica, obviamente, gerou um impacto também no contexto educacional, produzindo uma rápida transformação da população escolar.

\section{Políticas de educação intercultural}

Para enunciar os princípios que fundamentam uma dimensão intercultural na educação, devemos partir, sem dúvida, do texto da Constituição da República da Itália, aprovada pela Assembleia Constituinte no dia 22 de dezembro de 1947, que passou a vigorar no dia 1 de janeiro de 1948. Fazendo referência a valores laicos e igualitários no sistema educacional, a Constituição atribui à escola um verdadeiro papel de mediação dos conflitos sociais e culturais que ocorrem na sociedade.

De forma específica, na parte primeira, intitulada "direitos e deveres dos cidadãos", a Constituição aborda a questão da educação em dois artigos de grande relevância. O art. 33 estabelece a liberdade de ensino e o papel fundamental da República na instituição da escola:

A arte e a ciência são livres como livre é o seu ensinamento. A República dita as normas gerais sobre a instrução e institui escolas públicas para todos os níveis e graus. Entidades e particulares têm o direito de fundar escolas e institutos de educação, sem ônus para o Estado. A lei, ao fixar os direitos e as obrigações das escolas particulares que requerem a equiparação, deve assegurar plena liberdade às mesmas e, aos seus alunos, um tratamento escolar equivalente àquele dos alunos das escolas públicas. [...] As instituições de alta cultura, universidades e academias têm o direito de fixar ordenamentos autônomos nos limites determinados pelas leis do Estado

O Art. 34 estabelece, então, o princípio fundamental da inclusão educacional e, igualmente relevante, o direito ao apoio necessário no percurso de formação para aqueles que estão numa posição de dificuldade econômica:

A escola é aberta a todos. A instrução de primeiro grau, ministrada durante pelo menos oito anos, é obrigatória e gratuita. Os alunos capazes e aplicados, mesmo se carentes de meios econômicos, têm direito de atingir os graus mais altos de estudo. A República torna esse direito, mediante bolsas de

\footnotetext{
6 ISTAT. Indicatori demografici. Stime per I'anno 2014.

7 Sobre esta questão, cf. MIUR-ISMU. Alunni con cittadinanza non italiana. Rapporto nazionale a.s. 2013/2014. Tra difficoltà e successi.

8 Costituzione della Repubblica Italiana, 1947, art. 33.
} 
estudo, subsídios às famílias e outras medidas, que devem ser concedidas por concurso ${ }^{9}$.

O valor intercultural dessa perspectiva fica evidente na ideia delineada na Constituição de uma escola como um "instrumento de igualdade cívica, de respeito pela liberdade de todos os credos e de todas as opiniões ${ }^{10 "}$.

Com base nessas premissas, depois de uma longa tradição de acolhida das pessoas portadoras de necessidades especiais e de promoção da participação ativa das famílias durante os anos oitenta do século XX, a abordagem intercultural começa a se espalhar no sistema escolar italiano para fornecer respostas educativas à rápida mudança multicultural da sociedade ${ }^{11}$.

A primeira medida sobre o tema é a Circular Ministerial no 301, de 08 de setembro de 1989, Inserção de alunos estrangeiros no ensino obrigatório. Promoção e coordenação das atividades para o exercício do direito à educação, emanada com o objetivo de regulamentar o acesso geral ao direito à educação, à aprendizagem da língua italiana e à valorização da língua e da cultura de origem. Deve-se ressaltar que nesta fase o foco ainda está principalmente nos alunos estrangeiros, embora entre as orientações educacionais mais urgentes se sinalize que "encorajar os alunos a aceitar e a compreender [as] características [das diferentes culturas] contribui para promover uma consciência cultural aberta".

Uma abordagem mais consciente é expressa pela Circular Ministerial seguinte, n. 205, de 22 de julho de 1990, O ensino obrigatório e os alunos estrangeiros. A educação intercultural, com que se afirma pela primeira vez o princípio do envolvimento dos alunos italianos em uma relação interativa com os estudantes estrangeiros. Define-se também a educação intercultural como a forma mais alta e global de prevenção e de luta contra o racismo e todas as formas de intolerância. Mesmo na ausência de estudantes estrangeiros, as práticas educativas devem estar voltadas para impedir a formação de estereótipos contra pessoas e culturas.

Nos anos seguintes, há duas pronúncias importantes do Conselho Nacional da Educação: a primeira, em 23 de abril de 1992, sobre A educação intercultural nas escolas, em que são identificadas algumas necessidades do sistema de ensino em relação à orientação intercultural (entre elas a reforma dos currículos visando levar em conta as instâncias interculturais, a política para a formação inicial de professores de todos os níveis e graus escolares sobre estas questões, espaços institucionais, tempos e recursos suficientes para promover efetivamente a educação intercultural), e a segunda, de 24 de março de 1993, sobre o Racismo e

\footnotetext{
9 Ibidem, art. 34.

${ }^{10}$ CALAMANDREI, Piero. Difendiamo la scuola democratica, p. 90.

${ }^{11}$ Em relação ao percurso normativo da educação intercultural na Itália, cf. ONGINI, Vinicio. Buon compleanno intercultura! Una storia italiana lunga vent'anni, p. 104-110.
} 
o anti-semitismo hoje: o papel da escola, em que se reafirma, entre outras coisas, a necessidade de assumir os problemas da educação intercultural em uma visão sistêmica, apoiando a autonomia das escolas, com maior atenção à relação entre as "redes de escolas" e entre as diferentes autonomias, estabelecendo, ademais, a presença de mediadores interculturais e profissionais capazes de acompanhar o processo de integração dos estudantes estrangeiros.

Em 1994, como parte do processo de integração econômica e política em curso no contexto europeu, é emanada a Circular Ministerial n. 73, de 2 de março de 1994, O diálogo intercultural e a convivência democrática, que focaliza também as disciplinas e os programas educativos a serem reinterpretados em uma perspectiva intercultural.

Em 1997 é criada no Ministério da Educação a primeira "Comissão Nacional para a educação intercultural" para o aprofundamento das questões relacionadas à educação intercultural e para adoção das iniciativas necessárias para todos os tipos e graus de escola.

Em 1998 é emanada a primeira lei de imigração, a Lei n. 40, de 6 de março de 1998, que no seu artigo 36 enfatiza o valor educativo das diferenças linguísticas e culturais: "No exercício da autonomia didática e organizativa, as instituições de ensino realizam, para todos os alunos, projetos interculturais de ampliação da oferta de formação, visando à valorização das diferenças culturais e linguísticas e à promoção de iniciativas de acolhida e intercâmbio". Esse texto menciona, pela primeira vez, a necessidade de "mediadores culturais qualificados". O Decreto Legislativo seguinte, n. 286, de 25 de julho de 1998, Texto Único das disposições sobre a disciplina da imigração e normas sobre a condição do estrangeiro, reúne e coordena as várias disposições em matéria de imigração.

O Decreto do Presidente da República n. 394, de 31 de agosto de 1999, Regulamento com as normas de aplicação do Texto Único das disposições sobre a disciplina da imigração e normas sobre a condição de estrangeiro, deixa claro que cabe aos professores elaborar propostas para a distribuição de alunos estrangeiros nas aulas e definir, em relação aos níveis de competência dos alunos, a adaptação dos planos de ensino. Estabelece-se também que a inscrição de estudantes estrangeiros pode ocorrer a qualquer momento do ano letivo, a fim de proteger o direito fundamental à educação: “Crianças e adolescentes estrangeiros presentes no território nacional têm o direito à educação independentemente da regularidade da situação migratória, nas formas e condições previstas para os cidadãos italianos".

Em 2001 são promovidas ações de apoio com dinheiro adicional para o pessoal docente envolvido em escolas com expressiva incidência migratória, através da Circular Ministerial n. 155. 
Em 2002 é emanada a Lei de imigração n. 189, de 30 de julho de 2002, conhecida como "Bossi-Fini", que modifica a legislação anterior, adotando uma visão restritiva segundo a qual a legitimidade da presença de imigrantes no país está geralmente subordinada ao seu papel no mercado de trabalho.

Em 2006 é publicado um importante documento que manifesta certa maturidade sobre as questões da educação intercultural: a Circular Ministerial n. 24, de 1 de março de 2006, Orientações para a acolhida e a integração dos estudantes estrangeiros. Ao afirmar que "a Itália escolheu a plena integração de todos na escola e a educação intercultural como seu horizonte cultural", o documento oferece, em uma ótica pragmática, um manual concreto para enfrentar as dificuldades decorrentes da inclusão escolar de alunos de origem estrangeira, sublinhando que a educação intercultural é o ponto de referência a partir do qual se desenvolve a especificidade dos percursos formativos referentes a estudantes estrangeiros, no âmbito de uma ação educacional que abrange todos os alunos. Sensibilizando para o respeito das formas democráticas de convivência e oferecendo a oportunidade de adquirir conhecimentos históricos, sociais, jurídicos e econômicos indispensáveis para a formação da cidadania intercultural, a escola constitui um lugar privilegiado para a construção e a partilha de regras comuns.

Em outubro de 2007, é emitido o documento A via italiana para a escola intercultural e a integração dos estudantes estrangeiros, elaborado pelo Observatório nacional para a integração dos estudantes estrangeiros e para a educação intercultural, criado em dezembro de 2006 no Ministério da Educação.

A importância do texto está na definição de um "modelo de integração intercultural italiano". Na primeira parte do documento descrevem-se os princípios que inspiraram as melhores práticas interculturais realizadas na escola e a legislação a respeito:

- O universalismo, com a decisão de adotar critérios universalistas para o reconhecimento dos direitos das crianças e dos adolescentes, bem como a aplicação à realidade italiana das normas estabelecidas pela Convenção Internacional sobre os Direitos da Criança (aprovada pela ONU em 1989 e ratificada pela Itália em 1991), valorizando a tradição de inclusão da escola italiana, desenvolvida já na década de setenta do século passado, em relação às várias formas de diversidade;

- A escola comum, baseada na escolha de incluir alunos de cidadania nãoitaliana no interior das turmas escolares normais, evitando a construção de locais separados de aprendizagem, ao contrário do que aconteceu em outros países e em continuidade com orientações anteriores da escola italiana para a acolhida de várias formas de diversidade;

- A centralidade da pessoa em sua relação para com o outro, com a valorização da pessoa e a construção de planos educacionais inspirados na unicidade biográfica e relacional do estudante; 
- A intercultura, com a adoção de uma perspectiva orientada para a promoção do diálogo e do intercâmbio entre culturas, para todos os alunos, em todos os níveis: ensino, currículos, didática, disciplinas, relacionamentos, dia a dia das turmas.

A partir desses princípios, no documento são identificadas, a seguir, dez linhas de ação, que podem ser incluídas em três macro-áreas:

- Ações para a integração (1. Práticas de acolhida e inserção na escola; 2. Italiano como segunda língua; 3. Valorização do plurilinguismo; 4. Relações com as famílias estrangeiras e orientação);

- Ações para a interação intercultural (5. Relacionamentos na escola e no período extraescolar; 6 . Atividades referentes a discriminação e preconceitos; 7. Perspectivas interculturais nos conhecimentos e nas competências);

- Atores e recursos (8. A autonomia e as redes entre instituições de ensino, sociedade civil e território; 9. O papel dos administradores do sistema escolar; 10. O papel dos professores e do pessoal não docente).

A preocupação relativa aos contextos escolares com expressiva presença migratória caracteriza a emanação da controversa Circular Ministerial n. 2, de 8 de janeiro de 2010, que institui um limite máximo de 30\% para a presença de alunos com cidadania não-italiana nas turmas de toda ordem e grau. No mesmo período, amadureceu uma hipótese - no final rejeitada, mas discutida por muito tempo -, que previa "turmas de inserção" ou "turmas ponte" para a aprendizagem da língua italiana, ou seja, espaços de aprendizagem separados para os estudantes estrangeiros.

No modelo dos ENAF franceses (Enfants Nouvellement Arrivés en France), o foco se desloca para os alunos NAI (Neo Arrivati in Italia). Em 2008 foi assim elaborado um Plano nacional de ensino do italiano como segunda língua, para alunos de recente imigração matriculados em escolas secundárias de primeiro e segundo grau.

Em 2014, são emanadas, finalmente, novas Orientações para a acolhida e a integração dos estudantes estrangeiros (Circular Ministerial n. 4233, de 19 de fevereiro de 2014), em que se distingue entre várias tipologias de alunos de origem estrangeira (alunos sem cidadania italiana, sem ambiente familiar de língua italiana, menores não acompanhados, filhos de casais mistos, alunos que chegaram para a adoção internacional, alunos Roma, Sinti e Camminanti, estudantes universitários com cidadania estrangeira). O documento oferece orientações operacionais relativas à distribuição dos alunos estrangeiros nas escolas, sua acolhida, o envolvimento e a participação das famílias, a avaliação, a orientação, o ensino do italiano como segunda língua, as escolas com uma expressiva presença de estudantes estrangeiros, a formação do pessoal escolar e a educação dos adultos. 


\section{Estratégias e práticas de educação intercultural}

As rápidas mudanças na população escolar e as novas instâncias solicitadas pelos alunos com cidadania não-italiana levaram o sistema educacional a adotar, embora de forma muito heterogênea, abordagens e estratégias educativas direcionadas para a configuração intercultural do processo de aprendizagem ${ }^{12}$.

Nesse sentido, os dispositivos educacionais interculturais foram direcionados para dois propósitos essenciais:

- Por um lado, a inclusão dos estudantes estrangeiros (com ferramentas como o protocolo de acolhida, o protocolo de entrevista com os pais estrangeiros, o ensino do italiano como segunda língua, as iniciativas de peer education, as atividades dos mediadores interculturais, os serviços de informação e orientação para famílias estrangeiras);

- Por outro lado, a reestruturação mais abrangente da didática na perspectiva intercultural em benefício de todos os alunos (com abordagens tais como os percursos didáticos interculturais, a comissão para a intercultura, os processos de formação contínua do pessoal escolar, docente e não docente).

Algumas estratégias capazes de facilitar a construção de uma didática intercultural são, nesse sentido, os seguintes:

- A estruturação de espaços específicos e atividades para a acolhida de estudantes estrangeiros; este âmbito inclui os protocolos de acolhida, os materiais informativos multilíngues e os percursos de conhecimento para os pais estrangeiros sobre o sistema escolar italiano, inclusive mediante a presença de mediadores interculturais;

- A inicialização de processos de formação contínua do pessoal escolar, docente e não docente, sobre as questões da didática intercultural;

- A criação de uma "comissão intercultura", com funções de estímulo para a reorientação da didática na perspectiva intercultural (sem delegar poderes sobre a questão);

- A abertura, inclusive por meio de grupos de trabalho de professores, de uma reflexão crítica sobre os objetivos, conteúdos, métodos, instrumentos e estilos do processo de ensino-aprendizagem.

Na União Europeia, as diferentes estratégias para promover uma abordagem à educação intercultural foram agrupadas pela rede Eurydice nas seguintes tipologias principais:

12 Sobre estas questões, cf., entre outros, GOBBO, Francesca. Pedagogia interculturale. Il progetto
educativo nelle società complesse; DEMETRIO, Duccio, FAVARO, Graziella. Didattica interculturale.
Nuovi sguardi, competenze, percorsi; PORTERA, Agostino. Globalizzazione e pedagogia
interculturale. Interventi nella scuola; PINTO MINERVA, Franca. L'intercultura; ZOLETTO, Davide.
Straniero in classe. Una pedagogia dell'ospitalità; FIORUCCI, Massimiliano (a cura di). Una
scuola per tutti. Idee e proposte per una didattica interculturale delle discipline; ONGINI, Vinicio,
NOSENGHI, Claudia. Una classe a colori. Manuale per I'accoglienza e I'integrazione degli alunni
stranieri; SANTERINI, Milena. La qualità della scuola interculturale. Nuovi modelli per l'integrazione. 
- Medidas de orientação, que incluem informações por escrito sobre a escola, os mediadores interculturais, os recursos humanos e os instrumentais específicos, momentos de encontro para as famílias migrantes;

- Estratégias destinadas a reforçar a interação entre a escola e a família migrante, através da publicação de informações escritas sobre o sistema educativo nas línguas de origem dos estudantes estrangeiros e da utilização de mediadores interculturais ou professores de referência para a interligação entre os alunos migrantes, suas famílias e a escola;

- Ensino da língua materna, realizado geralmente em formas extracurriculares, com base em acordos bilaterais e na disponibilidade de recursos específicos;

- A promoção de processos mediante os quais as relações entre pessoas de diferentes origens culturais são analisadas e explicitadas nos currículos escolares, com uma finalidade intercultural que se configura em três níveis: a) a aprendizagem de valores de respeito e, em alguns casos, de anti-racismo, no contexto da diversidade cultural; b) a dimensão internacional, com um aprofundamento da diversidade cultural contemporânea nos contextos históricos e sociais; c) a questão da integração europeia ${ }^{13}$.

Além disso, de forma geral, os dispositivos de apoio aos alunos com um background migratório são adotados com base em dois modelos principais:

- Um modelo "integrado", no qual os estudantes estrangeiros são colocados em turmas "comuns". Neste caso, as medidas de apoio (essencialmente de natureza linguística) são fornecidas numa base individual para cada aluno durante o horário letivo normal;

- Um modelo "separado", que pode assumir duas formas: a) "disposições transitórias": os alunos estrangeiros são inseridos em grupos separados por um período de tempo limitado, durante o qual é proporcionado um apoio intensivo; b) "medidas de longo prazo": são formadas turmas especiais dentro da escola por um ou mais anos de escolaridade e, muitas vezes, os estudantes estrangeiros são agrupados de acordo com suas habilidades na língua de ensino.

Deve-se notar que o modelo denominado "separado" é pouco difundido na Europa e, quando presente, é usado em combinação com elementos do modelo "integrado". De fato, na maioria dos casos, há uma coexistência entre os dois modelos, preservando tanto quanto possível a inclusão na turma ordinária, com medidas de apoio linguístico transitórias ou prestadas com base na flexibilidade do horário didático. Em cerca de um terço dos países, ademais, são oferecidos aos estudantes estrangeiros cursos extracurriculares, fora do horário escolar oficial, na parte da tarde e durante o verão ${ }^{14}$.

\footnotetext{
${ }^{13}$ EURYDICE. Integrating Immigrant Children into Schools in Europe; EURYDICE. Integrating Immigrant Children into Schools in Europe: Measures to foster Communication with Immigrant Families and Heritage Language Teaching for Immigrant Children.

${ }^{14}$ Ibidem, p. 41-44.
} 
No contexto italiano, na atualidade, a inserção escolar dos estudantes estrangeiros está sendo realizada apenas no âmbito de um modelo "integrado". Esta escolha, de fato, corresponde a um modelo de educação inclusiva, que representa um valor irrenunciável para a construção de uma sociedade autenticamente intercultural.

\section{Conclusões}

Uma reflexão deve ser feita, em conclusão, em referência à necessidade de questionar criticamente a expressão - usualmente adotada na formulação de teorias, reflexões, hipóteses de trabalho no âmbito intercultural - de "estudantes estrangeiros" ou "com cidadania não-italiana". Se, por um lado, não devemos negar as necessidades específicas - de acolhida, orientação e aprendizagem de línguas - que caracterizam esses estudantes, precisamos também reconhecer que nem todos os estudantes estrangeiros manifestam as mesmas necessidades. De fato, as necessidades de um estudante recém-chegado em nosso país, por exemplo, são muito diferentes daquelas de um aluno que já frequentou por vários anos a escola italiana, ou daquelas de um jovem de segunda geração, nascido na Itália, ou que realizou em nosso país todo o percurso de primeira socialização. Além disso, apenas uma parte dos estudantes estrangeiros que frequentam as escolas italianas não é de língua italiana, ou seja, não domina o idioma italiano. Uma boa proporção deles é de segunda geração e não mostra, portanto, dificuldades de linguagem. Deverse-ia, portanto, superar a expressão "estudante estrangeiro", que é muitas vezes utilizada com ambiguidade, para desenvolver um raciocínio mais específico sobre as necessidades dos alunos, antes mesmo de atribuir-lhes conotações culturais.

A esse respeito, Milena Santerini utiliza a expressão "intercultura de segunda geração" como uma orientação consciente, que supera uma abordagem folclórica de exaltação da diferença cultural e que oferece estratégias culturais para combinar altos níveis de desempenho acadêmico para todos os alunos com o respeito das diferentes culturas, especialmente em contextos de presença expressiva de estudantes estrangeiros ${ }^{15}$.

Uma verdadeira perspectiva de educação intercultural requer também que, em âmbito escolar, sejam promovidos, de forma contínua, o diálogo e o intercâmbio entre culturas para toda a população escolar e para todos os níveis do processo de ensino-aprendizagem: no ensino, nos currículos, na didática, nas disciplinas, nos relacionamentos e no dia a dia da turma. Nesse sentido, a abordagem intercultural não se limita à promoção de meras estratégias de integração dos estudantes estrangeiros, mas visa o reconhecimento da diversidade como paradigma educacional, como uma oportunidade para valorizar todas as diferenças (de origem, gênero, classe social, percurso escolar).

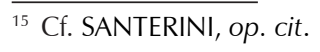


Dessa forma, o problema da integração de alunos de origem estrangeira passa pela luta contra a exclusão social de suas famílias. Por este motivo, é desejável uma maior interconexão entre as instituições escolares e o sistema de educação dos adultos, o que poderia facilitar a orientação dos pais dos alunos para percursos de aprendizagem permanente. Ainda são poucos, mas extremamente interessantes, os casos de escolas que assumem, mesmo através dos centros de educação dos adultos, a organização de percursos de aprendizagem do italiano para aqueles pais de estudantes estrangeiros que, por várias razões, não conseguem exercer seu direito à aprendizagem permanente.

Para enfrentar os problemas decorrentes dos contextos multiculturais parece necessário, então, apoiar as instituições escolares na construção de respostas territoriais sinérgicas com entidades públicas (autarquias locais, outras escolas, agências e instituições culturais públicas, bibliotecas públicas, serviços sociais e de saúde, autoridades locais para a educação, universidades) e privadas (associações culturais, instituições de caridade, ONGs, cooperativas, associações de imigrantes, clubes desportivos). Embora restrito a contextos limitados, esse tipo de respostas pode alcançar, de fato, resultados eficazes em termos de inclusão social.

\section{Bibliografia}

AA.VV. Manifesto della Razza. Il Giornale d'Italia, 4 luglio 1938.

BARN, Ravinder. Interculturalism in Europe: Fact, fad or fiction - the deconstruction of a theoretical idea. In AA.VV. Unedited workshop proceedings: Debating multiculturalism 1. London: Dialogue Society, 2012.

BEVILACQUA, Piero; DE CLEMENTI, Andreina; FRANZINA, Emilio (a cura di). Storia dell'emigrazione italiana. Vol. I - Partenze. Roma: Donzelli, 2001.

BEVILACQUA, Piero; DE CLEMENTI, Andreina; FRANZINA, Emilio (a cura di). Storia dell'emigrazione italiana. Vol. Il - Arrivi. Roma: Donzelli, 2002.

CALAMANDREI, Piero. Difendiamo la scuola democratica. In CALAMANDREI, Piero. Per la scuola. Palermo: Sellerio, 2008 (ed. or. 1950).

CAMPANI, Giovanna. Dalle minoranze agli immigrati. La questione del pluralismo culturale e religioso in Italia. Milano: Unicopli, 2008.

COLUCCl, Michele. Lavoro in movimento. L'emigrazione italiana in Europa, 1945-57. Roma: Donzelli, 2008.

Costituzione della Repubblica Italiana, 1947.

DEL BOCA, Angelo. Italiani in Africa Orientale. Vol. 1. Dall'Unità alla Marcia su Roma. Roma-Bari: Laterza, 1985.

DEL BOCA, Angelo. Italiani in Africa Orientale. Vol. 2. La conquista dell'Impero. Roma-Bari: Laterza, 1985.

DEL BOCA, Angelo. Italiani in Africa Orientale. Vol. 3. La caduta dell'Impero. RomaBari: Laterza, 1986.

DEL BOCA, Angelo. Italiani, brava gente? Un mito duro a morire. Vicenza: Neri Pozza, 2005. 
DEMETRIO, Duccio; FAVARO, Graziella. Didattica interculturale. Nuovi sguardi, competenze, percorsi. Milano: Franco Angeli, 2002.

EURYDICE. Integrating Immigrant Children into Schools in Europe. Brussels, 2004.

EURYDICE. Integrating Immigrant Children into Schools in Europe: Measures to foster Communication with Immigrant Families and Heritage Language Teaching for Immigrant Children. Brussels, 2009.

FIORUCCI, Massimiliano (a cura di). Una scuola per tutti. Idee e proposte per una didattica interculturale delle discipline. Milano: Franco Angeli, 2008.

GOBBO, Francesca. Pedagogia interculturale. Il progetto educativo nelle società complesse. Roma: Carocci, 2000.

GUNDARA, Jagdish. Interculturalism, Education and Inclusion. London: Paul Chapman, 2000.

ISTAT. Indicatori demografici. Stime per l'anno 2014. Disponível em: < http://www. istat.it/it/archivio/149003 > . Acesso em: 06.10.2015.

LABANCA, Nicola. Oltremare. Storia dell'espansione coloniale italiana. Bologna: II Mulino, 2007.

LORÉ, Michele. Antisemitismo e razzismo nella difesa della razza, 1938-1943. Soveria Mannelli: Rubettino 2008.

MINISTERO DELLA PUBBLICA ISTRUZIONE - OSSERVATORIO NAZIONALE PER L'INTEGRAZIONE DEGLI ALUNNI STRANIERI E PER L'EDUCAZIONE INTERCULTURALE. La via italiana per la scuola interculturale e l'integrazione degli alunni stranieri. Roma, 2007.

MIUR (MINISTERO DELL'ISTRUZIONE DELL'UNIVERSITÀ E DELLA RICERCA). Linee guida per l'accoglienza e l'integrazione degli alunni stranieri. Roma, 2014.

MIUR (MINISTERO DELL'ISTRUZIONE DELL'UNIVERSITÀ E DELLA RICERCA) - ISMU (ISTITUTO PER LO STUDIO DELLA MULTIETNICITÀ). Alunni con cittadinanza non italiana. Rapporto nazionale a.s. 2013/2014. Tra difficoltà e successi. Milano: Fondazione ISMU, 2015.

ONGINI, Vinicio. Buon compleanno intercultura! Una storia italiana lunga vent'anni. In CATARCl, Marco; FIORUCCI, Massimiliano (a cura di). Immigrazione e intercultura in Italia e in Spagna. Milano: Unicopli, 2011.

ONGINI, Vinicio; NOSENGHI, Claudia. Una classe a colori. Manuale per l'accoglienza e l'integrazione degli alunni stranieri. Milano: Vallardi, 2009.

PINTO MINERVA, Franca. L'intercultura. Roma-Bari: Laterza, 2007.

PORTERA, Agostino. Globalizzazione e pedagogia interculturale. Interventi nella scuola. Trento: Centro Studi Erickson, 2006.

PORTERA, Agostino. Pedagogia interculturale in Italia e in Europa. Milano: Vita e Pensiero, 1998.

SANTERINI, Milena. La qualità della scuola interculturale. Nuovi modelli per I'integrazione. Trento: Erickson, 2010.

ZOLETTO, Davide. Straniero in classe. Una pedagogia dell'ospitalità. Milano: Raffaello Cortina, 2007. 


\section{Abstract}

Intercultural education. Theories, policies and practices of cultural pluralism in the Italian education system

In the Italian context, educational research and intercultural practice move substantially along two main axes: a) in the first place, engaging in intercultural education means working to identify, design and test the most appropriate strategies for encouraging a positive insertion of foreign students in schools and, therefore, in society. This involves provision of the necessary conditions to ensure that all individuals (natives and immigrants) obtain the same rates of academic success; $b$ ) in the second place, because intercultural education is aimed at everyone and especially at native people, taking a perspective with an intercultural connotation means engaging in the objective of encouraging habits of reception in Italians. This can and must be reflected in the review and refoundation of the training axis of the school which must not aim only at the formation of the Italian citizen, but above all at the formation of a citizen of the world, who lives and acts in an interdependent global world.

Keywords: intercultural education, Italy, immigrant students, interculturalism, inclusion.

Recebido para publicação em 08.09.2015

Aceito para publicação em 05.11.2015

Received for publication in September 08 ${ }^{\text {th }}, 2015$

Accepted for publication in November 05 ${ }^{\text {th }}, 2015$

ISSN impresso 1980-8585

ISSN eletrônico 2237-9843

http://dx.doi.org/10.1590/1980-85852503880004609 